\title{
Facial palsy and Facial Muscle Spasm
}

\author{
MASUTO MISAWA M.D., Ph.D. \\ Shironishi Hospital \\ Matsumoto-shi, \\ Nagano-Ken, $\mathbf{T} 390$
}

JAPAN

\section{Anatomy}

The facial nerve is primarily motor nerve and includes partly the sensory nerve. In the outside of the skull, coming out first under the temporal from the stylo-mastoid foramen which is in the back of the styloid process, then between the mastoid process and the external auditory meatus, they run outside of the styloid process and the external carotid artery. And running in the parotid gland forwards, they are finally divided into the temporal branches, the zygomatic branches, the buccal branches, the mandibular branches or the cervical branches, and they govern its own motion.

As the fotegoing, the peripheries are connected with each other by the trigeminal nerve, the lingual nerve, the vagus nerve, the sympathetic nerve, the cervical nerve. Cause and Symptom...... (omitted)

\section{Therapy}

In order to stimulate the facial nerve directly, you have to make sure where is the top of mastoid process from the back of auricle and place the needles some $3.5 \mathrm{~cm}$ deep toward the external auditory foramen at the points about $0.5 \mathrm{~cm}$ forward of the top. When the needles touch the nerve without fail, the muscle spasm can be observed. If you feel uncertain, you had better move the needles a little for changing the depth or the direction.

When there's a difficulty of acupuncture in the facial nerve on the face, the facial muscle spasm can easily be seen without pains by applying the needles right in front of the lobe.

An ache means that the needles touch the trigeminal nerve, so you must change the places you are going to acupuncture. The same effect as you notice by inserting the needles into, the stylo-mastoid foramen can be observed by moving the needles in the forehead of the external auditory meatus along the back of mandible gradually down to the side. As for the facial spasm, the better results can be got by doing the same treatment for the facial palsy.

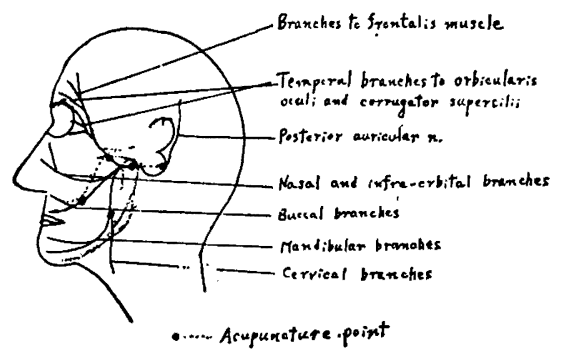

